\title{
Chloroaluminium phthalocyanine polymeric nanoparticles as photosensitisers: Photophysical and physicochemical characterisation, release and phototoxicity in vitro
}

\author{
Carina Silva de Paula ${ }^{\mathrm{a}, \mathrm{b}}$, Antonio Cláudio Tedesco ${ }^{\mathrm{c}}$, Fernando Lucas Primo ${ }^{\mathrm{c}}$, José Mário Carneiro Vilela ${ }^{\mathrm{d}}$, \\ Margareth Spangler Andrade ${ }^{\mathrm{d}}$, Vanessa Carla Furtado Mosqueira $\mathrm{a}, \mathrm{b}, \mathrm{e}, *$ \\ a Programa de Pós-Graduação em Nanotecnologia Farmacêutica, Universidade Federal de Ouro Preto, Campus Universitário Morro do Cruzeiro, Ouro Preto, MG 35400-000, Brazil

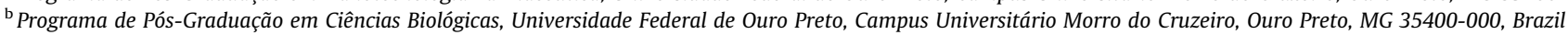

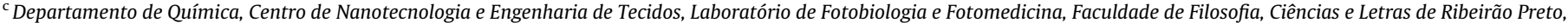 \\ Universidade de São Paulo, 14040-901 Ribeirão Preto-SP, Brazil \\ ${ }^{\mathrm{d}}$ Fundação Centro Tecnológico de Minas Gerais - CETEC, Avenida José Cândido da Silveira, 2000, Belo Horizonte, MG 31170-000, Brazil \\ e Departamento de Farmácia, Escola de Farmácia, Universidade Federal de Ouro Preto, Rua Costa Sena, 171 Centro, Ouro Preto, MG 35400-000, Brazil
}

\section{A R T I C L E I N F O}

Article history:

Received 28 June 2012

Received in revised form 17 March 2013

Accepted 20 March 2013

Available online 29 March 2013

\section{Keywords:}

Photodynamic therapy

Polymeric nanoparticles

Chloroaluminium phthalocyanine

Photophysical characterisation

Release kinetic

Phototoxicity in vitro

\begin{abstract}
A B S T R A C T
Nanoparticles of poly(D,L-lactide-co-glycolide), poly(D,L-lactide) and polyethylene glycol-blockpoly(D,L-lactide) were developed to encapsulate chloroaluminium phthalocyanine (AICIPC), a new hydrophobic photosensitiser used in photodynamic therapy (PDT). The mean nanoparticle size varied from 115 to $274 \mathrm{~nm}$, and the encapsulation efficiency ranged from 57\% to $96 \%$ due to drug precipitation induced by different types of polymer. All nanoparticle formulations presented negative zeta potential values ( $-37 \mathrm{mV}$ to $-59 \mathrm{mV}$ ), explaining their colloidal stability. The characteristic photophysical parameters were analysed: the absorption spectrum profile, fluorescence quantum yield and transient absorbance decay, with similar values for free and nanoparticles of AlClPc. The time-resolved spectroscopy measurements for AlClPc triplet excited state lifetimes indicate that encapsulation in nanocapsules increases triplet lifetime, which is advantageous for PDT efficiency. A sustained release profile over $168 \mathrm{~h}$ was obtained using external sink method. An in vitro phototoxic effect higher than $80 \%$ was observed in human fibroblasts at low laser light doses $\left(3 \mathrm{~J} / \mathrm{cm}^{2}\right)$ with $10 \mu \mathrm{M}$ of AlClPc. The AlClPc loaded within polymeric nanocapsules presented suitable physical stability, improved photophysical properties, sustained released profile and suitable activity in vitro to be considered a promising formulation for PDT.
\end{abstract}

(c) 2013 Elsevier B.V. All rights reserved.

\section{Introduction}

Photodynamic therapy (PDT) is an innovative and attractive modality for the treatment of oncologic disease (Allison and Sibata, 2010). The technique involves the topical or systemic administration of a photosensitiser (PS) that should concentrate more in tumour tissues than in normal tissues, followed by illumination of the tumour with visible light in a wavelength range matching the absorption spectrum of the photosensitiser (Allison and Sibata, 2010; Konan et al., 2002).

The resulting photodynamic reactions give rise to singlet oxygen $\left({ }^{1} \mathrm{O}_{2}\right)$ and to other active oxygen species that lead to tumour destruction. The PDT efficacy could be improved with the use of

\footnotetext{
* Corresponding author. Address: Escola de Farmácia-Universidade Federal de Ouro Preto, R. Costa Sena, 171 Centro CEP 35400-000 Ouro Preto, Minas Gerais, Brazil. Tel.: +55 31355910 32; fax: +55 3135591628 .

E-mail addresses: vamosqueira@gmail.com, mosqueira@ef.ufop.br (V.C.F. Mosqueira).
}

photosensitisers that strongly absorb red light above $650 \mathrm{~nm}$, where tissue exhibits optimal transparency. To this end, several new classes of potential sensitisers for PDT have been developed; among these, phthalocyanines have been found to be highly promising because of their high absorbance coefficient in the region of 650-680 nm (Konan et al., 2003; Sibata et al., 2004). Phthalocyanines can be chelated with a variety of metals, usually aluminium and zinc because these diamagnetic metals enhance their phototoxicity (Kluson et al., 2009).

Chloroaluminium phthalocyanine (AlClPc) is a photosensitiser with adequate photophysical properties for PDT (Nunes et al., 2004) (Fig. 1). Unfortunately, AlClPc is insoluble in water and biologically compatible solvents, which renders its systemic administration problematic and restricts possible medical applications (Nunes et al., 2004). To overcome this limitation, phthalocyanines have been associated to cyclodextrines (Silva et al., 2011) and to colloidal carriers such as liposomes (Nunes et al., 2004; Rocha et al., 2012), nanoemulsions (Rodrigues et al., 2012) and 


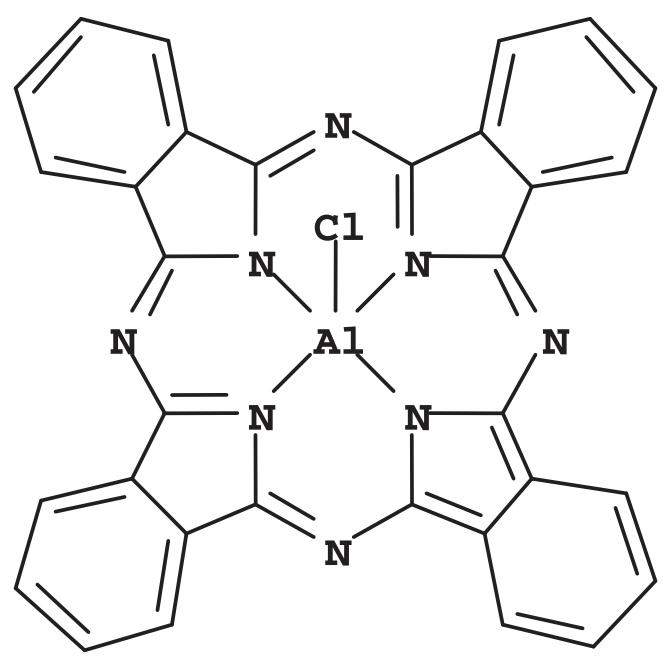

Fig. 1. Chemical structure of AlClPc.

nanocapsules (Moura-Siqueira et al., 2010; Oliveira et al., 2011). Polymeric nanoparticles (NPs) act as a drug vehicle to specifically target tumour tissues or cells in order to reduce the toxicity effect on normal tissue through the enhanced permeation and retention (EPR) effect (Maeda, 2010). Nanocapsules (NCs) are a carrier of choice for the intravenous administration of highly lipophilic drugs because they can dissolve or disperse drugs with high payload (Legrand et al., 1999). In NC, the oily nanodroplets are surrounded by a biodegradable polymeric wall that can be chemically modified to prolong blood circulation time or to improve the targeting properties of the carrier (Mosqueira et al., 2001b). To reduce undesirable uptake of NP by phagocytes, nanospheres (NSs) and NC were surface-modified with hydrophilic polymers such as polyethylene glycol (PEG) (Gref et al., 1994; Mosqueira et al., 2001a). The PEG corona on the NP surface decreased recognition by the MPS, which thereby increased the half-life of NC circulation in the blood (Mosqueira et al., 2001a, 2001b).

Based on these findings, the objective of this work was the development of formulations of AlClPc-loaded NC and NS prepared from different polymers. The AlClPc was associated with poly(D,Llactide-co-glycolide) (PLGA) or poly-D,L-lactide (PLA) to prepare conventional NC or NS and with the copolymer monomethoxypolyethylene glycol-co-poly-D,L-lactide (PLA-PEG) to prepare NC with prolonged blood circulation time. The effect of the formulation parameters on the photophysical and physicochemical properties was investigated. Furthermore, the influence of these variables on release kinetics and on phototoxicity in vitro was studied. This is the first report of the development of AlClPc photosensitiser in polymeric nanoparticles with a detailed investigation of its photophysical and physicochemical properties.

\section{Materials and methods}

\subsection{Drugs and reagents}

Chloro(29H,31H-phthalocyaninato)aluminium (AlClPc) was purchased from Aldrich Chemical Company Inc. (Milwaukee, WI, USA). Poly(D,L-lactide) (PLA, average Mw 42,000 g/mol) was provided by Phusis (France). Poly(D,L-lactide-co-glycolide) (85:15) (PLGA) and Poloxamer 188 (a non-ionic surfactant) were provided by Sigma-Aldrich (USA). PLA-PEG diblock copolymer (average Mw of $66,000 \mathrm{~g} / \mathrm{mol}$ with approximately $7.5 \%$ PEG at a Mw of $5000 \mathrm{~g} /$ mol) was provided by Alkermes (USA). Epikuron 170 (70\% soy phosphatidylcholine) was purchased from Lucas Meyer (France).
Miglyol 810N was a kind gift from Hulls (Germany). Polyethylene glycol (PEG 300) was provided by Synth (Brazil). Dimethylacetamide (DMA) was purchased from Vetec (Brazil). All of the solvents used were analytical grade, and other chemicals were commercially available reagent grade and were used without further purification. Milli- ${ }^{\circledR}$ water (Simplicity 185, Millipore, Bedford, USA) was used throughout. All experiments were carried out with stock solutions of AlClPc prepared in UV/HPLC-grade ethanol and stored in the dark at $4{ }^{\circ} \mathrm{C}$. The concentrations of phthalocyanine were assayed by HPLC (Waters Alliance 2695) with fluorescence detection (Waters 2475), using a $150 \mathrm{~mm} \times 4.6 \mathrm{~mm}, 4.6 \mu \mathrm{m}$ particle size C18 Gemini Phenomenex column protected by a Phenomenex security guard AJO $7597 \mathrm{C} 18$ column $(2 \mathrm{~mm} \times 4.6 \mathrm{~mm}, 3 \mu \mathrm{m})$ as the stationary phase.

\subsection{Preparation of AlClPc nanocapsules}

Conventional nanocapsules were obtained by interfacial polymer deposition following solvent displacement, which was previously described by Fessi et al. (1989) and was modified here to include AlClPc in the formulation. Conventional NC were prepared using the polymers PLA and PLGA. Briefly, $60 \mathrm{mg}$ of polymer was dissolved in $10 \mathrm{ml}$ of organic solution composed of $(0-70 \%)$ ethanol/acetone containing $75 \mathrm{mg}$ of Epikuron 170, $250 \mu \mathrm{l}$ of Miglyol $810 \mathrm{~N}$ and AlClPc $(0.1-0.2 \mathrm{mg} / \mathrm{ml})$. This organic solution was poured into $20 \mathrm{ml}$ of external aqueous phase containing $75 \mathrm{mg}$ of Poloxamer 188 under magnetic agitation. The solvents were evaporated under reduced pressure (Laborota 4000, Heidolph Instruments, Germany) until a final volume of $10 \mathrm{ml}$ was attained. Surface-modified NC were prepared using the diblock polymer of PLA-PEG without Poloxamer surfactant. Briefly, the polymer (75 mg) was dissolved in $10 \mathrm{ml}$ of organic solution composed of (0-70\%) ethanol/acetone containing $75 \mathrm{mg}$ of Epikuron 170 , $250 \mu \mathrm{l}$ of Miglyol $810 \mathrm{~N}$ and AlClPc $(0.1-0.2 \mathrm{mg} / \mathrm{ml})$. This organic solution was poured into the external aqueous phase $(20 \mathrm{ml})$. The solvents were evaporated under reduced pressure to a volume of $10 \mathrm{ml}$ (Mosqueira et al., 2001a). After $24 \mathrm{~h}$, the NC suspension was filtered through a $0.8 \mu \mathrm{m}$ sterile filter (Nalgene ${ }^{\circledR}$ ) to remove precipitated drug crystals that were not encapsulated. Nanospheres were prepared using the PLA polymer in the same way as described above but for the absence of Epikuron 170 and Miglyol 810 N.

\subsection{Nanocapsule characterisation}

\subsubsection{Zeta potential and size}

The mean size and size distribution of the NC were determined by photon correlation spectroscopy using an N5-Submicron Particle Size Analyser (Beckman Coulter, Florida, US). This method allows the determination of the mean diameter of the particle (hydrodynamic radius) and the polydispersity index (PI) of the particle population, which is a dimensionless measure of the broadness of the particle size distribution. Samples were analysed after appropriate dilution in ultra-pure Milli-Q water. The reported values were expressed as the means \pm standard deviations of at least three different batches of each nanocapsule formulation. The zeta potential was determined by Laser Doppler Anemometry (LDA) using the Zetasizer 3000 HS (Malvern Instruments, UK) after 250 -fold dilution of the colloidal suspension in $1 \mathrm{mM} \mathrm{NaCl}$. The conductivity of all NC dilutions was maintained close to $120 \mu \mathrm{S} /$ $\mathrm{cm}$. The measurements were performed at a constant $\mathrm{pH}$ of 7.1, as determined by the instrument.

\subsubsection{Atomic force microscopy (AFM)}

To examine the NC morphology, a $5 \mu$ l sample droplet was deposited on a freshly cleaved mica surface and then it was spread 
and dried by argon flow. The measurements were performed at room temperature and in air on a Dimension 3000 and with Multimode Equipment; both instruments were monitored by a Nanoscope IIIa controller from Digital Instruments (Santa Barbara, CA). The images were obtained in tapping mode using commercial silicon probes from Nanosensors with cantilevers of $228 \mu \mathrm{m}$ length, resonance frequencies of $75-98 \mathrm{kHz}$, spring constants of $3.0-$ $7.1 \mathrm{~N} / \mathrm{m}$ and a nominal tip radius of curvature of $8 \mathrm{~nm}$. The "scan rate" used was $1 \mathrm{~Hz}$. Dimensional analyses were carried out using the "section of analyses" application on the system. The values represent the mean \pm standard deviation derived from approximately 40 particle measurements.

\subsubsection{Determination of AlClPc encapsulation}

A determination of AlClPc content was performed using a methodology previously described and validated for the determination of chloroaluminium phthalocyanine in nanocarriers (Oliveira et al., 2011). The system consisted of HPLC (Waters Alliance 2695) with fluorescence detection (Waters 2475) using a $150 \mathrm{~mm} \times 4.6 \mathrm{~mm}$ and $4.6 \mu \mathrm{m}$ particle size C18 Gemini Phenomenex column protected by a Phenomenex security guard AJO 7597 C18 column $(2 \mathrm{~mm} \times 4.6 \mathrm{~mm}, 3 \mu \mathrm{m})$ as a stationary phase and methanol/acetone/dimethylformamide (90:5:15 v/v/v) as the mobile phase at a flow rate of $1 \mathrm{ml} / \mathrm{min}$. The samples were injected using the HPLC autosampler at an injection volume of $10 \mu \mathrm{l}$. The elution was monitored by a fluorescence detector via excitation and emission at $610 \mathrm{~nm}$ and $675 \mathrm{~nm}$, respectively, and the quantification limit was $1.12 \mathrm{ng} / \mathrm{ml}$.

The encapsulation yield in the nanoparticles was calculated by the difference between the total quantity of drug in the final colloidal suspension after filtration in a filter syringe (Filter syringe, $0.8 \mu \mathrm{m}$, Nalgene ${ }^{\circledR}$ ) minus the free drug in the external aqueous phase divided by the total quantity of drug in the colloidal suspension after filtration in the filter syringe $\times 100$ (Eq. (1)). The encapsulation yield, or drug loading, in the nanoparticles was determined from Eq. (1), where $A=$ concentration:

Encapsulation yield $(\%)=\frac{A^{\text {total }}-A^{\text {ultrafiltrate }} \times 100}{A^{\text {total in NP suspension }}}$

The encapsulation efficiency in the nanoparticles was calculated as the amount of drug truly encapsulated per ml divided by the total drug weight needed to prepare $1 \mathrm{ml}$ of NP formulation $\times 100$ (Eq. (2)). In this way, encapsulation efficiency takes into account the drug losses during the encapsulation process:

Encapsulation efficiency $(\%)=\frac{A^{\text {total }}-A^{\text {ultrafiltrate }} \times 100}{A^{\text {weight }}}$

where $A^{\text {total in NP suspension }}$ is the total quantity of drug in the final colloidal suspension after filtration in the filter syringe, $A^{\text {ultrafiltrate }}$ is the free drug in the external aqueous phase and $A^{\text {weight }}$ is the total drug weight needed to prepare the NP formulation.

The percentage of precipitated drug was calculated by the difference between the drug content/ml in the total suspension minus the drug content $/ \mathrm{ml}$ in the suspension after filtration by a $0.8 \mu \mathrm{m}$ filter divided by the drug content $/ \mathrm{ml}$ in the total suspension $\times 100$ as shown in Fig. 2.

Total AlClPc in the colloidal suspension was determined by full dissolution of $30 \mu \mathrm{l}$ in $970 \mu \mathrm{l}$ of ethanol/acetonitrile (1:1). The free drug that was soluble in the external aqueous phase was obtained by an ultrafiltration/centrifugation method wherein $400 \mu$ of the NC suspension was spun at $500 \times \mathrm{g}$ for $30 \mathrm{~min}$ in an AMICON device $\left(\right.$ Microcon $^{\circledR}$, molecular weight cut-off $=100,000$, Millipore $\left.{ }^{\circledR}\right)$. The amount of AlClPc bound to the ultrafiltration membrane was estimated by removing the membrane from the device. The membrane was rinsed with Milli-Q water, immersed in $500 \mu$ l acetonitrile, vortex-mixed for $15 \mathrm{~min}$ and centrifuged, and the supernatant was assayed for AlCIPc (Fig. 2).

\subsection{Spectroscopic and photophysical characterisations}

The NC samples were analysed for spectral absorption, fluorescence emission, time-resolved fluorescence and fluorescence quantum yield $\left(\phi_{f}\right)$, and an analysis of the transient absorption spectra and the AlClPc triplet excited-state lifetimes was performed.

\subsubsection{Absorption spectroscopy and fluorescence emission}

A known amount of AlClPc-loaded NC was extracted with dimethyl sulphoxide. Absorption measurements of the AlClPc extracted from NC and of AlClPc in ethanol were performed on a spectrophotometer (Perkin-Elmer Lambda 20) setup that scanned over the wavelength range from 300 to $800 \mathrm{~nm}$ and whose background was corrected using matched quartz cuvettes. Fluorescence emission spectra were performed on a Hitachi F-4500 spectrofluorimeter and a Fluorog 3 SPEX (Jobin Yvon, France) working at an excitation of $610 \mathrm{~nm}$, and fluorescence emission spectra were recorded between 650 and $750 \mathrm{~nm}$.

\subsubsection{Time-resolved fluorescence measurement $\left(\tau_{f}\right)$}

Time-correlated single-photon counting (TCSPC) was used to analyse the fluorescence lifetime $\left(\tau_{f}\right)$ of the AlClPc inside nanocapsules. The system used 2 banks of diode lasers emitting at $809 \mathrm{~nm}$ $(24 \mathrm{~W})$ to pump a solid-state laser crystal made of $\mathrm{Nd}: \mathrm{YVO}_{4}$ (Millenia Xs, Spectra Physics, Mountain View, California) with a fundamental emission wavelength of $1064 \mathrm{~nm}$. The laser beam then passed through a crystal that doubled the frequency, and the resulting beam had an intensity of $10 \mathrm{~W}$ with a $532 \mathrm{~nm}$ wavelength. This selected wavelength was used to irradiate a titanium-sapphire laser (Tsunami, Spectra Physics, Mountain View, California) that generated laser pulses (with 5 ps width) in the range of $840-1080 \mathrm{~nm}$. The maximum repetition frequency of the pulses was $82 \mathrm{MHz}$. This laser pulse frequency was divided by up to 8000 times, which gives a more appropriate frequency for the single photon count protocols. After the pulse selector, the beam passed through a third- and second-harmonic generator, which generated an output beam in the $280-330 \mathrm{~nm}$ wavelength range. The signal was detected by a setup board capable of measuring IRF (instrument response function) with a repetition rate of 60 ps. Software provided by the instruments was used to analyse the decay curves; the fit to an exponential decay was judged by inspection of the plots of the weighted residuals and by statistical parameters such as the reduced chi-square $\left(\chi^{2}\right)$.

\subsubsection{Fluorescence quantum yield $\left(\phi_{f}\right)$}

The fluorescence quantum yield $\left(\phi_{f}\right)$ of the AlCIPc was measured using the ratio method described by (Eaton, 1988) that utilised $\mathrm{ZnPc}$ in ethanol as a standard $\left(\phi_{f}=0.28\right)$ (Oliveira et al., 2005; Sibata et al., 2004). Optical densities were set below 0.1 a.u. for $\mathrm{NC}$ at the wavelength of absorbance $(610 \mathrm{~nm})$, and corrected fluorescence emission spectra between 650 and $750 \mathrm{~nm}$ were recorded on a Fluorog 3 spectrofluorimeter, following the technique described by Eaton (1988). Fluorescence quantum yield was calculated with the following equation:

$\phi_{u}=\left[\frac{A_{s} F_{u} n^{2}}{A_{u} F_{s} n_{o}}\right] \phi_{s}$

where $u$ marks unknown quantities, $s$ marks standard quantities, $\phi_{u}$ is the fluorescence quantum yield, $A$ is the absorbance, $F$ is the fluorescence area and $n$ is the refractive index. 


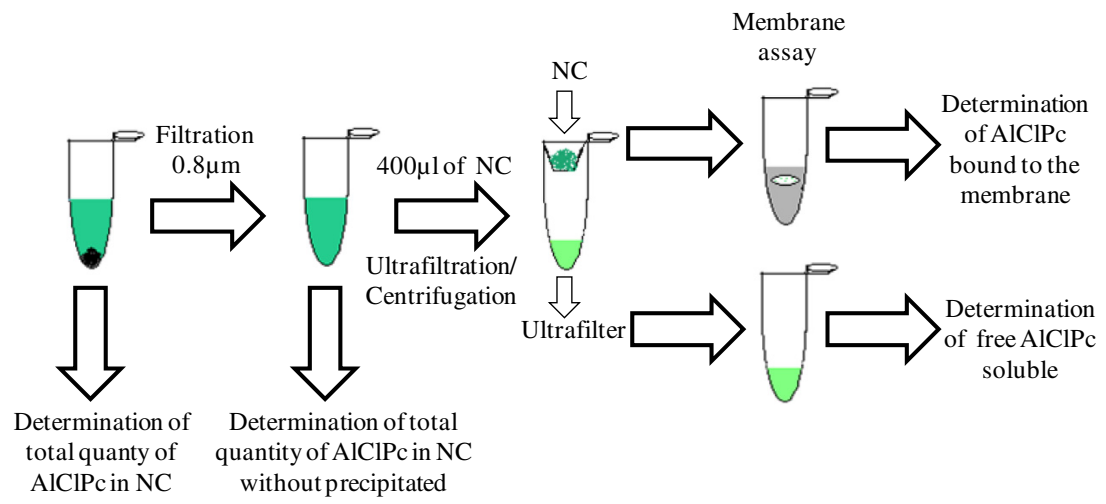

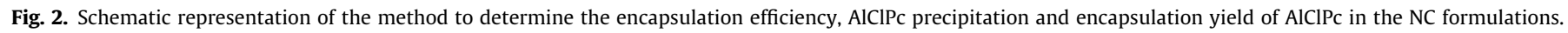

\subsubsection{Analysis of transient absorption spectra and AlClPc triplet excited state lifetimes}

The triplet state lifetime $\left(\tau_{T}\right)$ of AlClPc nanoparticles was calculated from a kinetics analysis of the mono-exponential decay for transient species obtained at the maximum absorption of these species at $480 \mathrm{~nm}$, as previously reported by Tedesco et al., 2003. The technique uses a laser flash photolysis spectrometer that allows the simultaneous capture of the transient absorption spectrum $(\lambda=300-800 \mathrm{~nm})$ and the transient kinetics at a single wavelength. The system used the third-harmonic $(355 \mathrm{~nm})$ of an Nd-YAG laser from Continuum (SURELITE I-10, Continuum, Santa Clara, California). The pulse length was $8 \mathrm{~ns}$, the diameter of the beam incident on the sample was $6 \mathrm{~mm}$ and the repetition rate was $10 \mathrm{~Hz}$. The pulse energy was $15 \mathrm{~mJ}$, as measured by a power metre (Field Master, Coherent, Santa Clara, California). A tungsten-halogen lamp ( $400 \mathrm{~W}$ ) was used as a probe. The lamp beam was collimated (1 $\mathrm{mm}$ in diameter) through the sample in the cell holder, which was held in a $1 \mathrm{~cm}$ cuvette. The growth-decay kinetics were measured at a single wavelength using a monochromator M300 and a photomultiplier R928P from Benthon Instruments (Livingston, UK) Transient decays were averaged using a digital oscilloscope (TDS 340A, Tektronix, Wilsonville, Oregon). The stored, digitised kinetic decays were analysed with software supplied by Edinburgh Analytical Instruments (Livingston, UK).

\subsection{Solubility and in vitro release studies}

The solubility of AlClPc in n-octanol was determined at room temperature. An excess of free $\operatorname{AlClPc}(5 \mathrm{mg})$ was equilibrated with the $n$-octanol $(500 \mu \mathrm{l})$ for $24 \mathrm{~h}$ under vortex agitation (Vortex Instrument, IKA, Germany). Then, the supernatant was centrifuged at $800 \mathrm{~g}$ for $30 \mathrm{~min}$ and filtered in a filter syringe (Filter syringe, $0.45 \mathrm{~m}$, Nalgene $\left.{ }^{\circledR}\right)$, and aliquots $(5 \mu \mathrm{l})$ were taken and analysed by HPLC. The analysis was performed in triplicate.

Release kinetic studies were carried out in two different media: phosphate buffered saline (PBS) and $n$-octanol. A theoretical sink condition (10\% of the saturation concentration) was maintained in the $n$-octanol medium. The NP suspensions were first filtrated in a filter syringe (Filter syringe, $0.8 \mu \mathrm{m}$, Nalgene ${ }^{\circledR}$ ) to remove any precipitated drug.

The direct dialysis method was used for AlClPc release studies in PBS. Aliquots of $1.0 \mathrm{ml}$ of NP suspension containing $0.2 \mathrm{mg} / \mathrm{ml}$ of AlClPc were enclosed in dialysis bags (cellulose membrane, Mw cut-off of 12,400, Sigma) and incubated in $30 \mathrm{ml}$ of PBS (pH $7.4)$ at $37^{\circ} \mathrm{C}$ under mild agitation in a water bath $(n=3)$. At predetermined time intervals, $500 \mu \mathrm{l}$ samples were withdrawn from the incubation medium and analysed for AlClPc with HPLC, as described above. After sampling, the incubation medium was replaced by fresh PBS.

The external sink method was chosen for the AlClPc release studies because AlClPc has extremely low solubility in water and this medium ( $n$-octanol) is capable of providing perfect sink conditions (Chorny et al., 2002). Thus, aliquots of $0.5 \mathrm{ml}$ of NS and NC aqueous suspension containing $0.2 \mathrm{mg} / \mathrm{ml}$ of AlClPc were put in a centrifuge tube with $1.5 \mathrm{ml} n$-octanol. The tubes were immersed in a water bath at $37^{\circ} \mathrm{C}$ under agitation. At each prescribed time interval, three tubes were taken out and centrifuged at $8000 \mathrm{rpm}$ for $5 \mathrm{~min}$. The organic layer was withdrawn and transferred into a test tube for analysis. A control experiment to determine the release behaviour of the free drug was also performed. The amount of drug released was determined by HPLC with fluorescence detection as previously cited in Section 2.3.3.

\subsection{Assay of in vitro phototoxicity}

Human fibroblasts were allowed to grow to confluence in Dulbecco's Modified Eagle Medium (DMEM) with glucose (Gibco ${ }^{\circledR}$ ) without glutamine and supplemented with $10 \%(\mathrm{v} / \mathrm{v})$ foetal bovine serum (Cultilab) and antibiotics. Cells were detached from plates with $0.05 \%$ trypsin $\left(\mathrm{Gibco}^{\circledR}\right)$. Fibroblasts were seeded into 24-well plates at $5 \times 10^{6}$ cells/ml ( $1 \mathrm{ml}$ per well) and allowed to grow for $24 \mathrm{~h}$ in an incubator $\left(5 \% \mathrm{CO}_{2}, 37^{\circ} \mathrm{C}\right.$ and humidified atmosphere). On the day of the experiment, the culture medium was removed. A different culture medium containing some formulation of AlClPc nanoparticles at a final concentration of $10 \mu \mathrm{M}$ of AlClPc was added to each well. Free AlClPc ( $1 \mathrm{mg}$ ) was dissolved in $1 \mathrm{ml}$ of DMA/PEG $300(2: 3)$ and diluted 80-fold in cell culture medium to render a $10 \mu \mathrm{M}$ concentration (Krishna et al., 1993). Cells were incubated for $4 \mathrm{~h}$ at $37{ }^{\circ} \mathrm{C}$ in a $5 \% \mathrm{CO}_{2}$ atmosphere. In the photocytotoxicity assay, the DMEM was removed and DMEM without Phenol Red was added before irradiation. Irradiation was performed with a diode laser (Eagle Quantum-tech) equipped with an optical fibre $\left(750 \mathrm{~mW}\right.$ and $140 \mathrm{~mJ} / \mathrm{cm}^{2}, 3.0 \mathrm{~J} / \mathrm{cm}^{2}$ or $10.0 \mathrm{~J} / \mathrm{cm}^{2}$ ) under sterile conditions. Cell viability was measured immediately after irradiation by determining mitochondrial activity using a colorimetric MTT assay according to the method described in Mosmann (1983). Optical densities on the microplates were determined at $540 \mathrm{~nm}$ using a Safire2-TECAN instrument. The dark toxicity (no light exposure) was also determined.

\subsection{Statistics}

All experiments were performed in triplicate and were expressed as mean values \pm standard deviations. Mean sizes, zeta potentials and drug release data at each time point were compared 
by an ANOVA test using the Epilnfo 6.04 program, and a probability of $5 \%$ was considered to be significant.

\section{Results and discussion}

\subsection{Nanocapsules characterisation}

The nanoparticles were prepared by the modified solvent displacement method first reported by Fessi et al. (1989). This preparation technique offers considerable advantages in the preparation of nanodevices for intravascular delivery due to the use of excipients of low toxicity and non-chlorinated solvents that can be easily removed from the preparation (Fessi et al., 1989). Furthermore, it produces nanometric carriers with a suitably narrow distribution of sizes using biodegradable polymers such as PLA (Legrand et al., 2007). The obtained NC showed high AlClPc concentration in the final colloidal suspension $(0.2 \mathrm{mg} / \mathrm{ml})$ when considering the low solubility in water of AlClPc (Oliveira et al., 2011). Typically, a NC has an oily core in which lipophilic drugs can be dissolved or dispersed and which is surrounded by a polymeric biodegradable wall (Legrand et al., 1999). In the present work, the AlClPc encapsulation yield was close to $100 \%$. The amount of free AlClPc dissolved in the aqueous phase of the colloidal suspension or adsorbed onto the ultrafiltration membrane was not able to be quantified because it was lower than the quantification limit of the method (Oliveira et al., 2011) (Table 1).

The amount of ethanol incorporated in the organic phase during the preparation of the NC alters the encapsulation efficiency of the nanoparticles (Labib et al., 1991 and Chorny et al., 2002). Because the drug is highly soluble only in absolute ethanol, it is likely that the ethanol concentration significantly altered drug partition between the phases. Table 1 shows that the encapsulation efficiency was higher when the formulations of PLGA NC, PLA NC and PLAPEG NC were prepared with $70 \%$ ethanol in organic phase $(p<0.05)$. These results are in agreement with the data obtained by Labib et al. (1991) for nanocapsules of ZnPc. Moreover, the formulations of PLA-PEG NC and PLA NC prepared with 70\% ethanol presented a smaller \% of precipitate drug than those prepared with $50 \%$ of ethanol in the organic phase $(p<0.05)$.

When PLGA was used as the polymeric wall of the NC, the \% of precipitate was increased because the PLGA has lower solubility in ethanol (Table 1). However, the amount of non-precipitated AlCIPC increases in encapsulation efficiency because AlClPc has good solubility in ethanol. An increase in ethanol concentration is also responsible for reduction of the size of the PLGA NC $(p<0.05)$, which is most likely influenced by the rapid precipitation of polymeric nanovesicles during the diffusion of both solvents. As the formation of nanoparticles is kinetically controlled in the nanoprecipitation method, this fast precipitation of polymers prevents particle aggregation and keeps particles in a lower size range (Labib et al., 1991 and Chorny et al., 2002). This effect was not observed with PLA and PLA-PEG $(p<0.05)$, which are more soluble in ethanol.

Increases in drug concentration produced a reduction in size and increased the drug association with the nanocarrier for PLAPEG and PLGA. A possible explanation for this is an interaction of the AlClPc with the polymeric wall on the NC surface to produce surface active complexes. This interaction can also be observed in the release profile of NC, shown in Fig. 7, in which a burst effect was obtained for the PLGA and PLA-PEG formulations but not for PLA nanocapsules. In the case of PLA, these complexes are most likely formed to a lower extent, and the largest amount of AlClPc is associated with the oily core and increases in size as drug concentration increases $(p<0.05)$. This is in accordance with the lower encapsulation efficiency obtained for PLA nanospheres because of the absence of an oily nucleus (Table 1 ). These results indicate that the oily core of an NC can dissolve or disperse hydrophobic molecules with higher payload, as has been previously discussed by Legrand et al. (1999).

Particle sizes must be strictly controlled in nanoparticulate formulations intended for intravascular delivery. Because the estimated diameter of the smallest blood capillaries in the human body is in the 4-7 $\mu \mathrm{m}$ range (Kreuter, 1996), the particle size should optimally be kept in the submicron range to prevent occlusion of capillaries. The formulations of AlClPc loaded in NP show a mean polydispersity index (P.I.) of $0.128 \pm 0.02$. PI is used to judge the sample's quality; a P.I. near 0.1 indicates that samples have excellent quality with a narrow size distribution. The P.I. values for the different NP formulations are shown in Table 1.

The NC prepared in this study show negative $\zeta$ potential values ranging from -59.6 to $-37.2 \mathrm{mV}$. The absolute mean values of $\zeta$ potential for the PLA NC and PLGA NC were not significantly modified with increases in drug concentration (Table 1$)(p>0.05)$ in either type of nanoparticles. This most likely occurs due to the effect of surface masking provided by the Poloxamer surfactant, which is present in the PLA and PLGA NC formulations (Mosqueira et al., 2000). However, an increase of the AlClPc concentration in formulations of PLA-PEG NC from 0.1 to $0.2 \mathrm{mg} / \mathrm{ml}$ decreased the absolute mean value of $\zeta$ potential $(p<0.05)$ because Poloxamer is absent. One possible explanation for this is an interaction of

Table 1

Characterisation of AlClPc nanoparticles obtained from different polymers.

\begin{tabular}{|c|c|c|c|c|c|c|c|}
\hline $\begin{array}{l}\mathrm{NP} \\
\text { formulation }\end{array}$ & $\begin{array}{l}\text { AlClPc (mg/ } \\
\mathrm{ml})\end{array}$ & $\begin{array}{l}\text { Ethanol } \\
(\%)\end{array}$ & $\begin{array}{l}\text { Encapsulation } \\
\text { efficiency (\%) }\end{array}$ & $\begin{array}{l}\text { AlClPc precipitate } \\
(\%)\end{array}$ & $\begin{array}{l}\text { Mean size } \pm \text { SD } \\
(\mathrm{nm})^{\mathrm{b}}\end{array}$ & $\mathrm{PI}^{\mathrm{c}}$ & $\begin{array}{l}\zeta \text { potential } \pm \text { SD } \\
(\mathrm{mV})^{\mathrm{d}}\end{array}$ \\
\hline \multirow[t]{3}{*}{ NC PLGA } & 0.1 & 70 & $84.3 \pm 3.9$ & $9.2 \pm 0.6$ & $226.6 \pm 2.6$ & 0.120 & $-55.4 \pm 3.7$ \\
\hline & 0.1 & 50 & $76.0 \pm 0.3$ & $2.7 \pm 0.3$ & $273.7 \pm 3.0$ & 0.095 & $-54.7 \pm 3.0$ \\
\hline & 0.2 & 50 & $95.6 \pm 2.0$ & $4.3 \pm 2.0$ & $178.0 \pm 0.1$ & 0.116 & $-50.7 \pm 1.4$ \\
\hline \multirow[t]{3}{*}{ NC PLA } & 0.1 & 70 & $80.9 \pm 2.0$ & $2.0 \pm 2.0$ & $180.3 \pm 0.6$ & 0.143 & $-51.9 \pm 4.2$ \\
\hline & 0.1 & 50 & $59.5 \pm 2.0$ & $8.8 \pm 0.6$ & $182.9 \pm 2.0$ & 0.116 & $-50.7 \pm 1.4$ \\
\hline & 0.2 & 50 & $89.8 \pm 1.5$ & $5.3 \pm 1.7$ & $198.9 \pm 2.3$ & 0.141 & $-50.5 \pm 0.8$ \\
\hline \multirow[t]{3}{*}{ NC PLA-PEG } & 0.1 & 70 & $87.5 \pm 1.4$ & $0.13 \pm 0.1$ & $207.0 \pm 1.9$ & 0.144 & $-59.6 \pm 2.0$ \\
\hline & 0.1 & 50 & $66.5 \pm 0.6$ & $17.3 \pm 1.3$ & $189.6 \pm 0.1$ & 0.126 & $-47.9 \pm 3.8$ \\
\hline & 0.2 & 50 & $73.6 \pm 2.0$ & $7.1 \pm 3.4$ & $138.6 \pm 0.7$ & 0.126 & $-37.2 \pm 4.1$ \\
\hline NS PLA & 0.2 & 50 & $56.7 \pm 0.4$ & $10.0 \pm 2.7$ & $115.3 \pm 2.5$ & 0.148 & nd \\
\hline
\end{tabular}

nd $=$ Not determined. The percentage of loading was $100 \pm 0.1$ for all formulations.

a Ethanol \% in organic phase of the formulations.

b Standard deviation $(\mathrm{n}=3)$ of the population reported by the instrument.

c Polydispersity index.

d $n=3$ Measurement after dilution 1:250 in 1 mmolar $\mathrm{NaCl}$. 
the AlCIPc with the polymeric wall in PLA-PEG NC, which decreases the absolute value of the mean zeta potential because PEG chains in PLA-PEG polymer show low "PEG coating efficiency" as discussed previously by Vila et al. (2004). Furthermore, the influence of AlClPc on reducing the size of PLA-PEG NC can play an important role in the zeta potential values because the surface potential increases as AlClPc interactions with the NC wall increase; this can reduce the absolute value of the mean zeta potential. This hypothesis is reinforced by the burst effect in the PLA-PEG release profile observed in Fig. 7. Another influencing factor is the amount of ethanol used in the NC preparation process, which significantly $(p<0.05)$ altered the zeta potential of PLA-PEG NC. Ethanol most likely interferes in the PEG's conformational arrangement (solvation effect) at the NC surface in PLA-PEG NC, which influences the zeta potential by providing lower surface charge shielding as previously reported by Mosqueira et al. (2001a). This effect could explain the increase in surface charge with increasing ethanol content.

The morphology of the nanoparticles was examined by AFM. The NC suspensions appeared as a homogeneous population of particles in AFM images (Fig. 3A and B). The AFM allows direct measurement of the size of the NP in samples deposited on freshly cleaved mica plates, and it permits simultaneous characterisation of particle shape and stiffness. The PLA-PEG NC external morphological analyses (shown in Fig. $3 \mathrm{~A}$ ) revealed that all nanostructures were spherical, had a regular surface and presented a halo surrounding the internal core, which is evidenced by a different texture in the phase image (right side). The NC presented a homogeneous distribution in height and in three-dimensional images (Fig. 3A and B). An analysis of AFM data indicates that the sizes of PLA NC and PLA-PEG NC decreased with drug loading. These differences were significant $(p<0.05)$. Analysis of the data indicates that the diameter of the nanocapsules is much larger than the height (Table 2) for all NC formulations (Fig. 4). The PLA nanospheres' diameter/height ratio was 1.5 , which was calculated from the topographical profile of the AFM images (Fig. 4 and Table 2). NS are generally harder than nanocapsules. These results are attributed to the flattening of the NC on a mica surface (Leite et al., 2005) due to the liquid nature of the core. NP stiffness was estimated by the diameter/height ratio (Table 2 ). The results show that association of AlClPc with NP has no significant effect $(p>0.05)$ on NP stiffness. The same can be observed with the polymeric wall (PLA or PLA-PEG) for those preparations. Only the NP type (NC or NS) affects significantly the flattening of the NP.

\subsection{Photophysical characterisation}

The absorption spectra of an AlClPc standard is compared to that of AlClPc extracted from NC in dimethyl sulphoxide in Fig. 5. In all NC preparations, AlClPc exhibits a strong absorbance in the red region with a maximum wavelength at $676 \mathrm{~nm}(670 \mathrm{~nm}$ in ethanol) as shown in Table 3. Furthermore, it presents a group of $Q$ bands in the region from 600 to $710 \mathrm{~nm}$ and a Soret band at $340 \mathrm{~nm}$. Notably, incorporation of the drug into a NC causes a redshift in these bands. The incorporation of dyes into micro-heterogeneous media produces redshifts, which is in agreement with previously reported work (Sibata et al., 2004). As shown in Fig. 5 the absorption spectrum profile for the AlCIPC in NC was identical to the spectrum observed for AlClPc in ethanol. In all NC formulations, the results indicate the absence of dimeric AlCIPc or its aggregation states.

The fluorescence emission spectrum shows a maximum emission for AlClPc at $679 \mathrm{~nm}$ in ethanol and $680 \mathrm{~nm}$ in NC after light excitation at $610 \mathrm{~nm}$ (Fig. 6). A redshift in the fluorescence maximum is observed due to the environmental change from homogeneous (ethanol) to heterogeneous media, in this case the NC. The redshift observed for the encapsulated phthalocyanines is a minimal value $(<10 \mathrm{~nm})$. Generally, this phenomenon is related to the drug-polymer interaction from non-bonded intermolecular forces.

The single photon counting technique was used to determined fluorescence lifetime for AlClPc in ethanol and in NC (Table 3). Singlet excited-state lifetimes were determined through the decay curve profiles of AlClPc in NC and in organic media. Mono-exponential decay was observed for the AlClPc standard in ethanol and for AlCIPC in PLGA NC, PLA NC and PLA-PEG NC, which indicates that the AlClPc is distributed into one site (or population) with one singlet excited-state lifetime. The AlClPc in NC presented lifetimes of 5.77, 5.80 and 5.85 ns for PLGA NC, PLA NC and PLAPEG NC, respectively (Table 3 ).

The relationship between emitted and absorbed photons results in a fluorescence quantum yield $\left(\phi_{f}\right)$ as calculated by Eq. (1). The fluorescence quantum yield for the AlClPc standard and for the NC formulations are similar (Table 3), which indicates that the photosensitiser does not suffer degradation during the encapsulation. The fluorescence quantum yield of the formulations was evaluated using ZnPc in ethanol as a standard $\left(\phi_{f}=0.28\right)$ (Oliveira et al., 2005; Sibata et al., 2004). The fluorescence quantum yields obtained for AlClPc in ethanol were $0.81,0.72$ and 0.80 for PLGA NC, PLA NC and PLA-PEG NC, respectively. The value of the fluorescence quantum yield is directly related to the photodynamic efficiency of the photosensitiser. Values near unity indicate that the fluorophores are more efficient as fluorescence probes for diagnostic use than as photosensitisers for PDT. The fluorescence quantum yield of AlClPc (0.8) was higher than that of ZnPc (0.28), which indicates it can be usefully applied to photo-diagnosis procedures. However, the compound also has an appropriate oxygen quantum yield in the homogeneous medium $\left(\phi_{\Delta}=0.3\right)$ as described by Idowu and Nyokong (2007). This characteristic is an important factor and is essential to produce PDT-effectiveness based on the tumour inactivation mechanisms (Type I/II).

The transient absorbance spectra for the drugs were obtained by laser flash photolysis, and triplet lifetimes $\left(\tau_{T}\right)$ were calculated from a kinetic analysis of the transient decays (Table 3 ). The triplet excited-state absorbed in the wavelength range from 380 to $650 \mathrm{~nm}$ with a maximum transient absorption at $470 \mathrm{~nm}$ for AlClPc in ethanol and AlClPc in NC. Beyond the triplet absorption, groundstate photobleaching of the Q and Soret bands of the AlClPc during the excitation process can be observed (data not shown). The decay curve adjustments were performed according to the chi-square function. The obtained transient absorbance spectra showed the presence of transient species that decayed mono-exponentially with characteristic lifetimes and resulted in significantly higher lifetimes for the triplet state of AlClPc in NC (Table 3). The triplet state reacts with forms of molecular oxygen $\left(\mathrm{O}_{2}\right)$ by an energy transfer process leading to singlet oxygen, which is the key agent in cell damage in PDT. Therefore, the longer the triplet excited state lifetime, the better is the probability that drug energy can be transferred to molecular oxygen and, consequently, to produce singlet oxygen.

\subsection{Solubility and in vitro release studies}

The AlClPc was found to be practically insoluble in water at room temperature and in PBS at $\mathrm{pH} 7.4$ (the amount detected was lower than $1.12 \mathrm{ng} / \mathrm{ml}$ ). In the release study of AlClPc from PLA NC and PLA-PEG NC in PBS, the AlClPc concentrations in the external medium were below the detection limit by HPLC $(<1.12 \mathrm{ng} / \mathrm{ml})$. Sink conditions could not be obtained in PBS and water media, which is explained by the very low solubility of the drug in water and by the tendency of AlClPc to self-aggregate in aqueous media. Phthalocyanines display a strong tendency to form dimers in water due to their large hydrophobic skeleton, which 


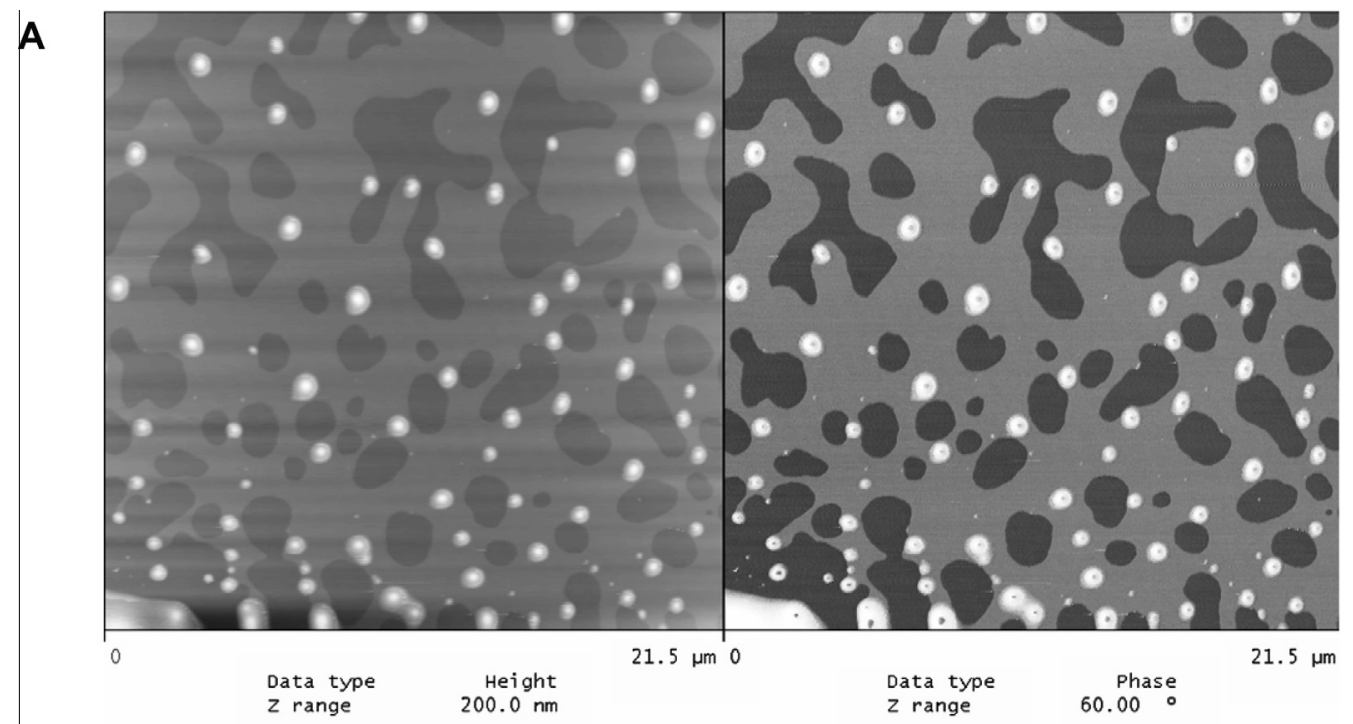

B

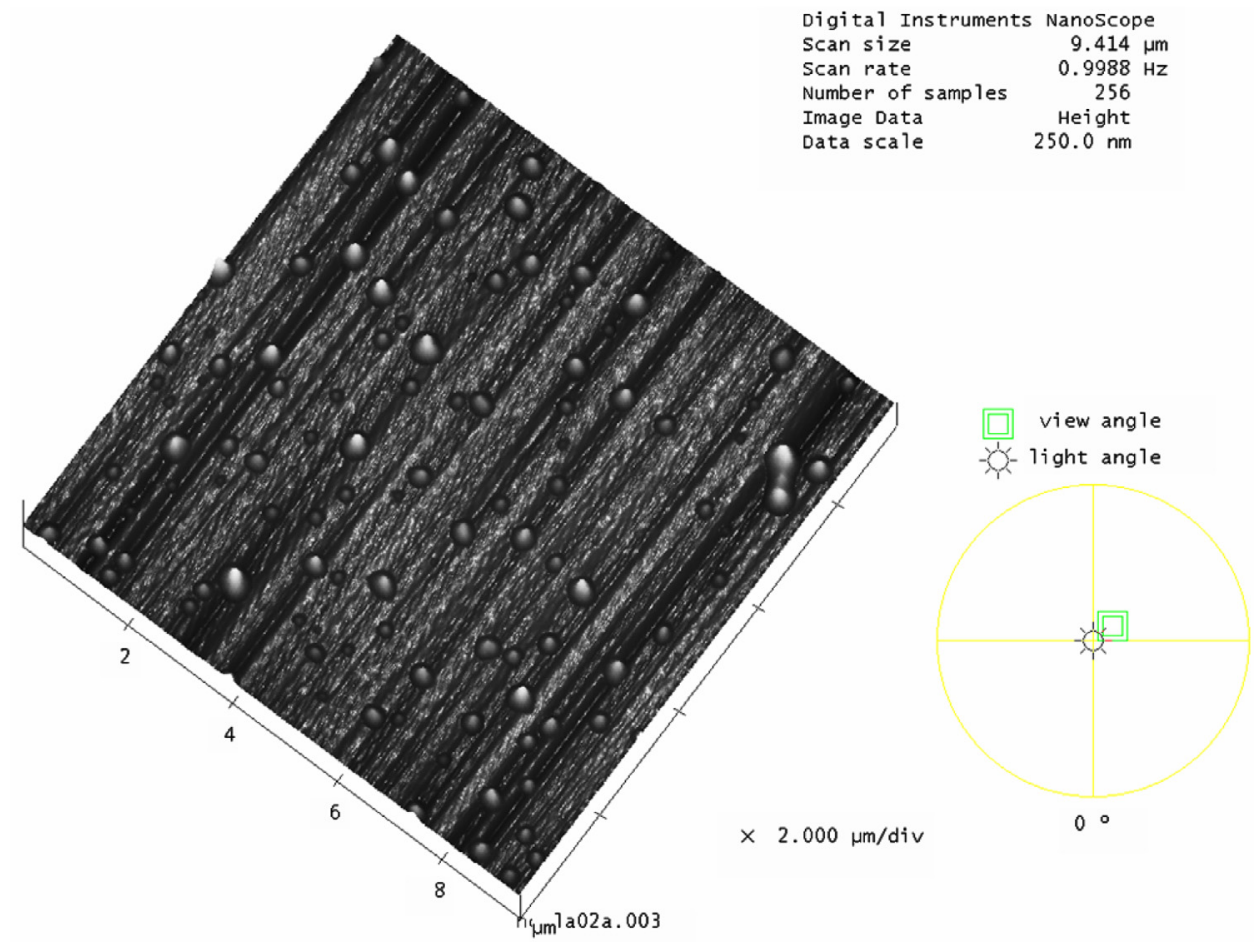

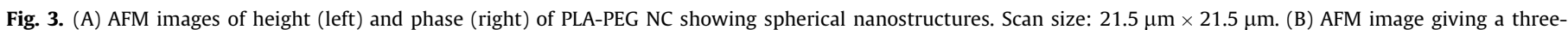
dimensional view of PLA NC containing AlClPc $(0.2 \mathrm{mg} / \mathrm{ml})$ spread on mica. Scan size: $10 \mu \mathrm{m} \times 10 \mu \mathrm{m}$.

avoids contact with the aqueous medium (Dhami and Phillips, 1996). The release study using the direct dialysis method results in no detectable AlClPc in the external medium.

Therefore, the external sink method was used to study the in vitro release of AlClPc using $n$-octanol as described by Chorny

Table 2

Characterisation of the nanoparticles by Atomic Force Microscopy.

\begin{tabular}{llll}
\hline $\begin{array}{l}\text { Nanoparticles } \\
\text { formulation }\end{array}$ & $\begin{array}{l}\text { AlClPc }(\mathrm{mg} / \\
\mathrm{ml})\end{array}$ & $\begin{array}{l}\text { Mean size } \pm \text { SD } \\
(\mathrm{nm})^{\mathrm{a}}\end{array}$ & $\begin{array}{l}\text { Ratio diameter/ } \\
\text { height }\end{array}$ \\
\hline PLA NC & 0.0 & $265.3 \pm 37.5$ & 8 \\
AlClPC-PLA NC & 2.0 & $211.8 \pm 31.4$ & 11 \\
PLA-PEG NC & 0.0 & $270.7 \pm 68.3$ & 11 \\
AlClPc-PLA-PEG NC & 2.0 & $153.5 \pm 49.9$ & 8 \\
PLA NS & 2.0 & $98.0 \pm 25.2$ & 1.5 \\
\hline
\end{tabular}

a Standard deviation $(n=40)$. et al. (2002). The solubility of AlClPc in $n$-octanol at room temperature was $1.2 \mathrm{mg} / \mathrm{ml}$. This method is not sensitive enough to study rapid release formulations, but can be used to release formulations having a long release time (Soppimath et al., 2001). $N$-octanol was established as the external medium of choice due to the similarity between its lipophilicity and the lipophilicity of biological membranes (Chorny et al., 2002).

Fig. 7 shows the in vitro drug release profiles of free AlClPc and AlClPc-loaded PLA NS, PLA NC, PLA-PEG NC and PLGA NC in the first 7 days. These in vitro release studies demonstrated that these types of drug carriers allow for extended delivery of the drug over more than 1 week (Fig. 7) compared to the solubility profile of free AlClPc. The analysis of the AlClPc release profile from PLGA NC shows approximately $10 \%$ released in a burst in the first $6 \mathrm{~h}$ followed by a slow release over 7 days that does not exceed $18 \%$. A 

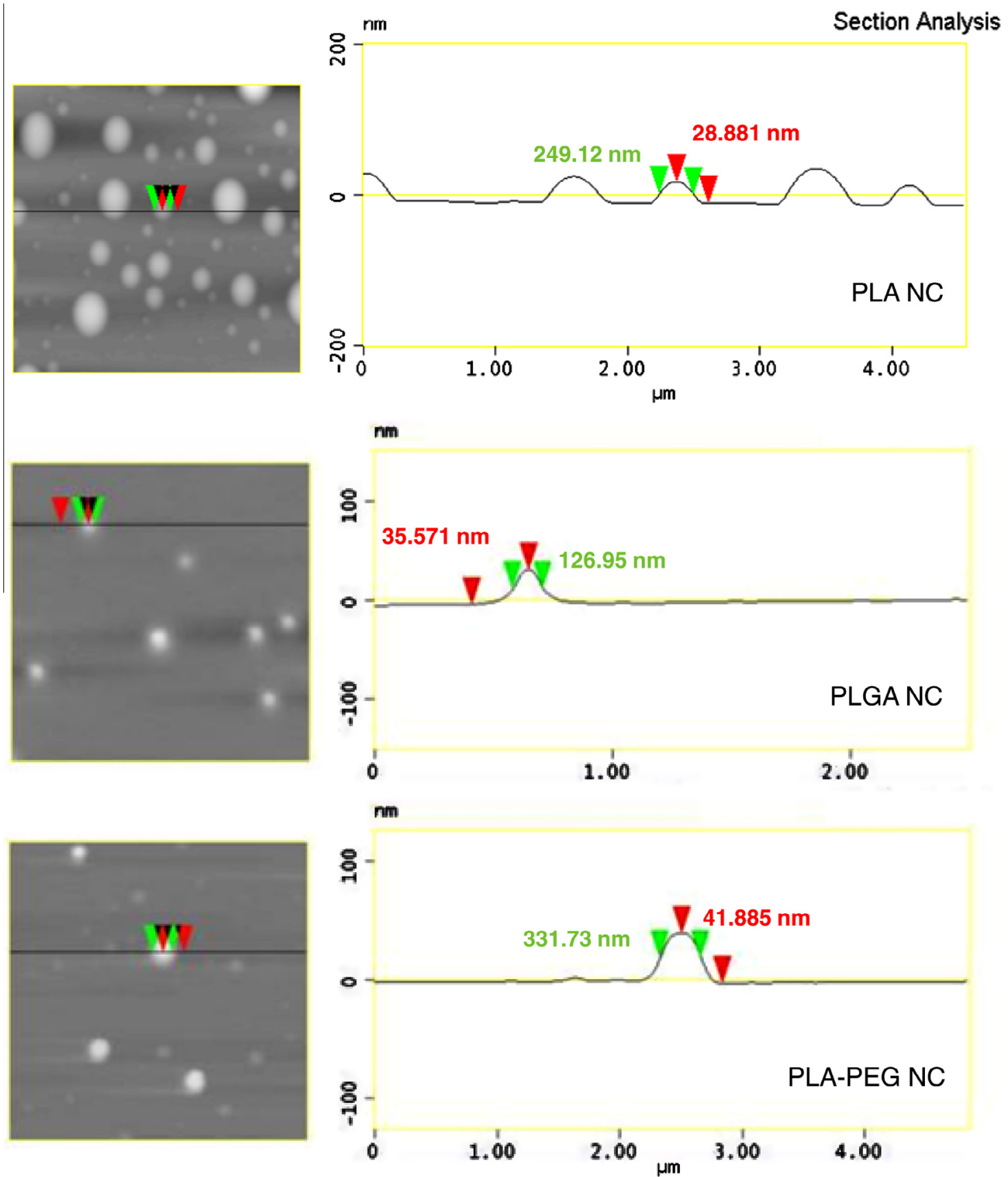

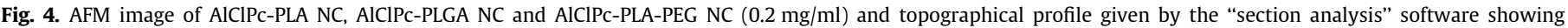
measurements of nanocapsule diameter and height.

good association of AlClPc with PLGA NC, which was also evidenced by the encapsulation efficiency, seems to explain this profile. The AlCIPc releases from PLA NC and PLA-PEG NC displayed an initial burst of $14.7 \%$ and $18.5 \%$ up to $2 \mathrm{~h}$, respectively. These effects were followed by the slow release of $52.5 \%$ and $39.1 \%$ after 7 days for PLA NC and PLA-PEG NC, respectively. After the burst effect, the release of all $\mathrm{NC}$ formulations follows a first-order release profile. The initial burst may be caused by AlClPc molecules associated to the surface of the NC, particularly with PLA-PEG NC. Zeta potential data (Table 1) corroborate this hypothesis. The release of AlCIPc from the PLA NS also involved an initial rapid release phase that was followed by a phase of relatively slow release. Approximately $55 \%$ of the AlClPc was released after 7 days from the nanospheres (Fig. 7). On the other hand, when free AlClPc was incubated with the acceptor media, 35.7\% was rapidly dissolved in the first 120 min. Thus, more than $70 \%$ of the free AlClPc was dissolved in $n$-octanol over 7 days (Fig. 7 ) in contrast to the $55 \%$ released from PLA nanospheres over that time. Entrapment of AlClPc in nanoparticles, particularly in PLGA and PLA-PEG, significantly prolongs its in vitro release $(p<0.05)$.

\subsection{Assay of in vitro phototoxicity}

Photodynamic therapy requires three components to be present simultaneously for cytotoxicity: a sensitiser, light and oxygen. In this study, the in vitro phototoxicity of AlClPc using different formulations of NP was compared. The phototoxic effect was observed in human fibroblasts in the presence of laser light doses 


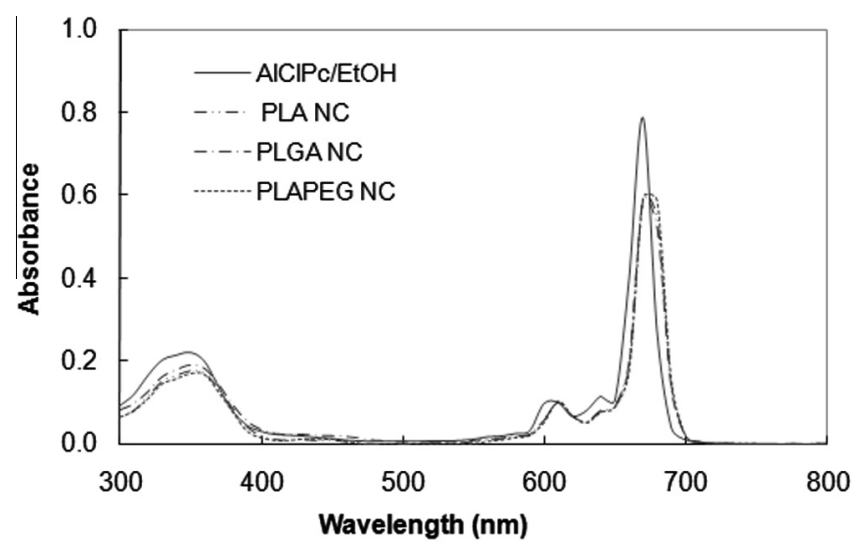

Fig. 5. Absorption spectra of AlClPc in ethanol: (-) AlClPc standard, (-.-.-) AlClPc in PLGA NC, (-..-) AlClPc in PLA NC and (-----) AlCIPC in PLA-PEG NC.

of $140 \mathrm{~mJ} / \mathrm{cm}^{2}, 3 \mathrm{~J} / \mathrm{cm}^{2}$ and $10 \mathrm{~J} / \mathrm{cm}^{2}$ at AlClPc concentrations of $10 \mu \mathrm{M}$. Fibroblasts are an excellent biological model to evaluate the biocompatibility of new biomaterials and innovative pharmaceutical formulations. First, it must be noted that light alone did not result in a significant decrease in cell viability under the experimental conditions used for this study (data not shown). The cytotoxicity of free AlClPc and unloaded and AlClPc-loaded NC and NS were also evaluated in the dark.

No significant cytotoxicity in the dark was observed after treatment with free AlClPc and unloaded or AlClPc-loaded PLA NC, PLA NS, PLA-PEG NC and PLGA NC formulations $(p>0.05)$. As shown in Fig. 8, cellular viability was not significantly affected after treatment with unloaded PLA NC, PLA NS, PLA-PEG NC and PLGA NC in the absence of light $(p>0.05)$. This is consistent with the known biocompatibility and safety of PLA and PLGA polymers (Davda and Labhasetwar, 2002; Konan et al., 2003). EMT-6 cell viability was not affected, for example, after treatment with drug-free PLA and PLGA nanoparticle formulations with irradiation at $9 \mathrm{~J} / \mathrm{cm}^{2}$ (Konan et al., 2003).

Fig. 9 shows the in vitro cytotoxic effect of free AlClPc and loaded-NP at light doses of $140 \mathrm{~mJ} / \mathrm{cm}^{2}, 3 \mathrm{~J} / \mathrm{cm}^{2}$ and $10 \mathrm{~J} / \mathrm{cm}^{2}$ and AlClPc concentrations of $10 \mu \mathrm{M}$. After irradiation at $140 \mathrm{~mJ} / \mathrm{cm}^{2}$, all formulations were effective at inducing cell damage irrespective of the delivery vehicle as demonstrated by their similar phototoxicity; there were no significant differences among them $(p>0.05)$. The photoactivity of AlClPc-loaded NP was compared to the free AlClPc solution after irradiation at a light dose of $140 \mathrm{~mJ} / \mathrm{cm}^{2}$ (Fig. 9). The viabilities of treated cells with free AlClPc, PLA NC, PLA NS, PLGA NC and PLA-PEG NC containing AlClPc and a light dose of $140 \mathrm{~mJ} / \mathrm{cm}^{2}$ were reduced to $58.7 \%, 51.3 \%, 41.3 \%, 57.5 \%$
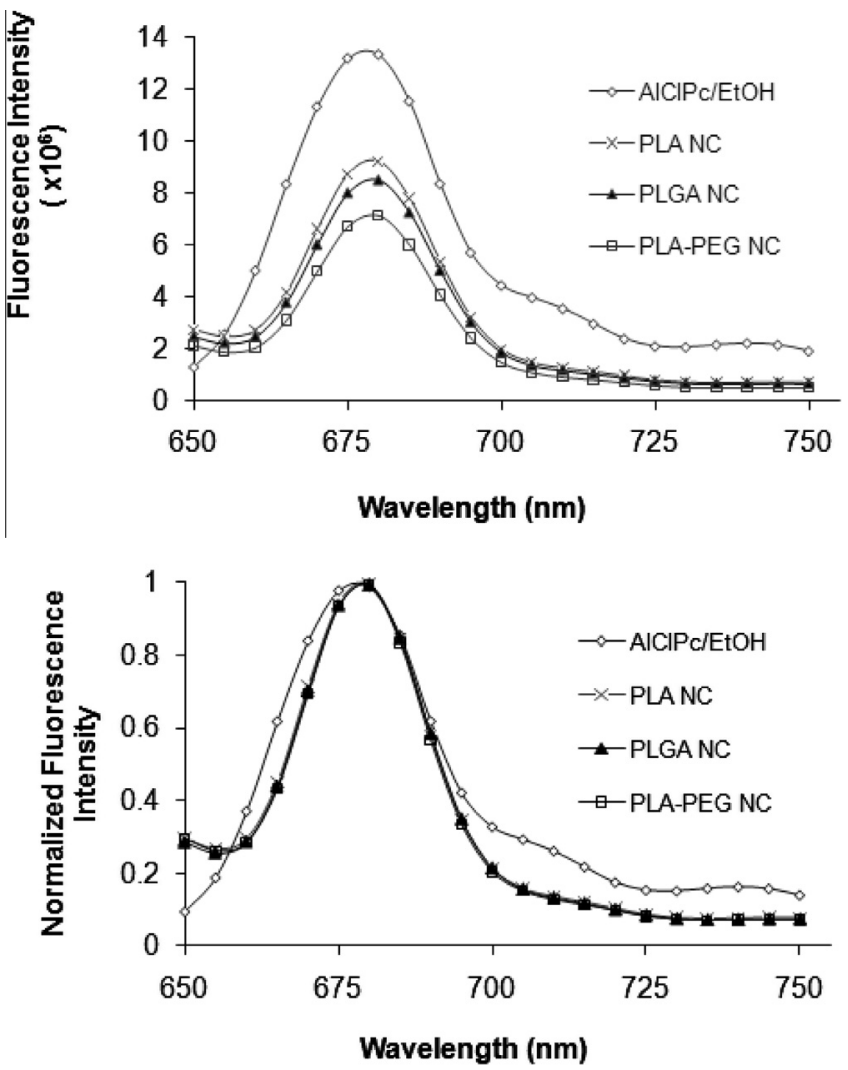

Fig. 6. Fluorescence emission spectra of AlCIPc in $(\diamond)$ ethanol, in $(\boldsymbol{\Delta})$ PLGA NC, in $(\times)$ PLA NC and in ( $\square$ ) PLA-PEG NC.

and $46.4 \%$, respectively. There was no significant difference between free AlClPc and AlClPc-loaded NP at $140 \mathrm{~mJ} / \mathrm{cm}^{2}(p>0.05)$.

The cells treated with free AlClPc demonstrated a reduction of cell viability proportional to an increase of dose of light $(p<0.001)$. Increasing the dose to $3 \mathrm{~J} / \mathrm{cm}^{2}$ with free AlClPc solution reduced the cell viability to $33.3 \%$; the value was only $15 \%$ at $10 \mathrm{~J} /$ $\mathrm{cm}^{2}$ (Fig. 9). At the same time, free AlClPc presented similar $(p>0.05)$ phototoxicity to all NP formulations at $140 \mathrm{~mJ} / \mathrm{cm}^{2}$. AlClPc-loaded nanoparticles at $3 \mathrm{~J} / \mathrm{cm}^{2}$ were more efficient at inducing cellular death than free AIClPc $(p<0.001)$. This may be explained by the interaction of the NP with cells, which would result in a high AlClPc association to cells and improved PDT efficiency.

Concerning cells treated with NP formulations, there were no significant differences $(p>0.05)$ between the values of cellular viability at laser light doses of $3 \mathrm{~J} / \mathrm{cm}^{2}$ or $10 \mathrm{~J} / \mathrm{cm}^{2}$ based on the

Table 3

Photophysical characterisation.

\begin{tabular}{|c|c|c|c|c|c|c|c|}
\hline Samples & $\mathrm{UV} \mathrm{vis}^{\mathrm{a}}(\mathrm{nm})$ & $\lambda_{\mathrm{EX}}{ }^{\mathrm{b}}(\mathrm{nm})$ & $\lambda_{\mathrm{EM}}{ }^{\mathrm{c}}(\mathrm{nm})$ & $\tau_{f}^{\mathrm{d}}(\mathrm{ns}) \pm \mathrm{SD}$ & $\tau_{f}^{\mathrm{e}}(\%)$ & $\phi_{f}{ }^{\mathrm{f}}$ & $\tau_{T}^{\mathrm{e}}(\mu \mathrm{s})^{\mathrm{g}} \pm \mathrm{SD}$ \\
\hline Free AlClPc & 670 & 610 & 679 & $7.57 \pm 0.06$ & 100 & 0.81 & $0.80^{\mathrm{h}}$ \\
\hline AlClPc loaded PLGA NC & 676 & 610 & 680 & $5.77 \pm 0.09$ & 100 & 0.81 & $1.38 \pm 0.05$ \\
\hline AlClPc loaded PLA NC & 676 & 610 & 680 & $5.80 \pm 0.09$ & 100 & 0.72 & $1.37 \pm 0.03$ \\
\hline AlClPc loaded PLA-PEG NC & 676 & 610 & 680 & $5.85 \pm 0.08$ & 100 & 0.80 & $1.41 \pm 0.06$ \\
\hline
\end{tabular}

a Maximum wavelength UV-vis absorption.

b Excitation wavelength.

c Maximum wavelength fluorescence emission.

d Fluorescence lifetimes, mean $\pm \mathrm{SD}$.

e Population distribution.

${ }^{\mathrm{f}}$ Fluorescence quantum yield using $\mathrm{ZnPc}\left(\phi_{f}=0.28\right)$ in ethanol as a standard.

$\mathrm{g}$ Triplete lifetime, mean $\pm \mathrm{SD}(n=3)$.

h Nunes et al., 2004. 


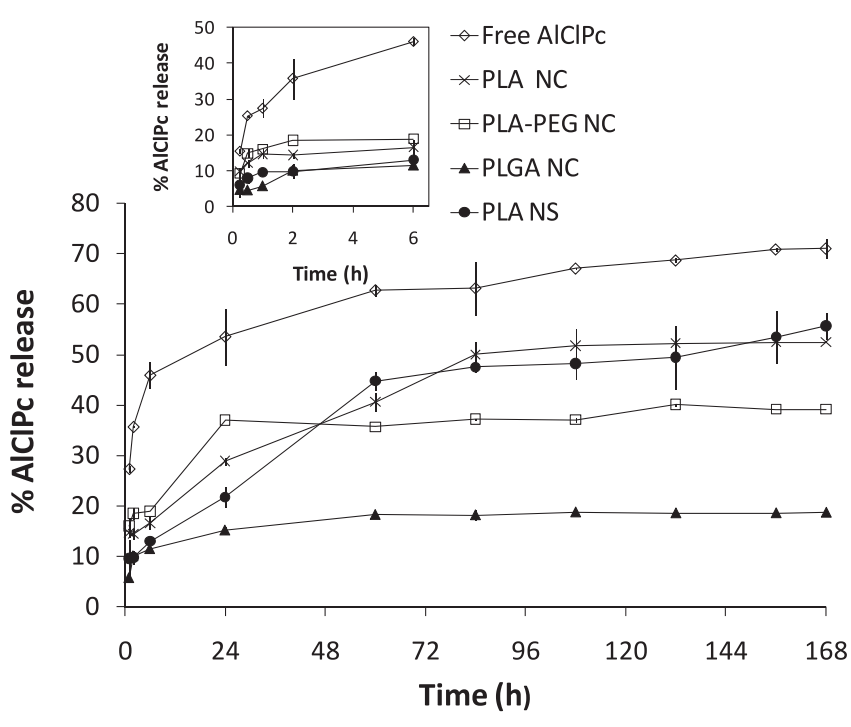

Fig. 7. In vitro drug release profile of $(\diamond)$ free AlClPc and AlClPc loaded in $(\bullet)$ PLA NS, ( $\Delta)$ PLGA NC, $(\times)$ PLA NC and ( $\square)$ PLA-PEG NC. The insert graph represents the in vitro drug release profile of AlCIPc during the first $6 \mathrm{~h}$. The results are the means of three experiments \pm SD.

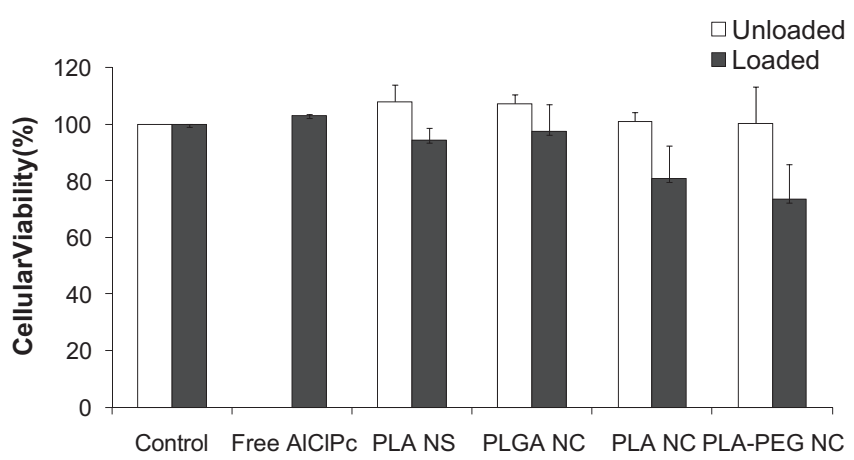

Fig. 8. Cytotoxicity of free AlClPc $(10 \mu \mathrm{M})$ and unloaded and AlClPc-loaded NP in the dark ( $10 \mu \mathrm{M}$ of AlClPc in the nanoparticle form) in human fibroblast culture. Cell viability is expressed as the mean $\pm \operatorname{SD}(n=3)$.

mitochondrial activity test (MTT) (Fig. 9). The viabilities of treated cells with AlClPc-loaded PLA NC, PLA NS, PLGA NC and PLA-PEG NC that were irradiated at a light dose of $3 \mathrm{~J} / \mathrm{cm}^{2}$ were $12.9 \%, 15.5 \%$, $13.7 \%$ and $11.3 \%$, respectively. After irradiation at a dose of $10 \mathrm{~J} /$ $\mathrm{cm}^{2}$, the viabilities of incubated cells with PLA NC, PLA NS, PLGA NC and PLA-PEG NC containing AlClPc were reduced to $13.5 \%$, $15.2 \%, 18.4 \%$ and $13.5 \%$, respectively (Fig. 9). The results analysis indicates that no significant difference occurs in the phototoxic effect observed for AlClPc incorporated into different formulations $(p>0.05)$ under higher doses of light. The dose of $3 \mathrm{~J} / \mathrm{cm}^{2}$ was enough to drastically reduce the cell viability by activating the AICIPC photosensitiser in all NP formulations. Kolarova et al. (2007) reported the production of reactive oxygen species (ROS) and assessed the phototoxicity of disulphonated chloroaluminium phthalocyanine (ClAlPcS2) using G361 human melanoma cells. They showed that the optimum phototoxic effect observed in G361 melanoma cells was obtained for a combination of a laser dose of $25 \mathrm{~J} / \mathrm{cm}^{2}$ and $5-10 \mathrm{mg} / \mathrm{ml}$ of ClAlPcS2. Those combinations of sensitiser concentration and corresponding radiation dose were lethal for the melanoma cells. The results obtained herein showed that much lower doses of AlClPc and light were necessary to reduce

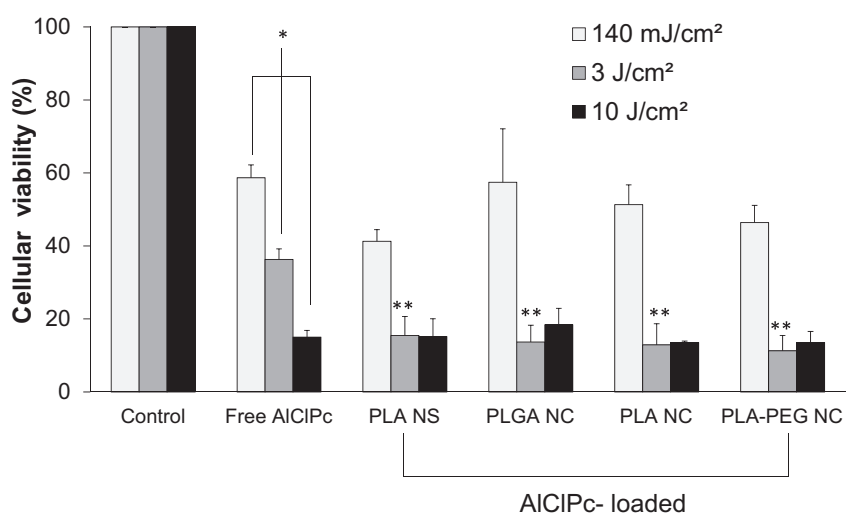

Fig. 9. AlClPc phototoxicity in different nanoparticles irradiated with $140 \mathrm{~mJ} / \mathrm{cm}^{2}$ $3 \mathrm{~J} / \mathrm{cm}^{2}$ and $10 \mathrm{~J} / \mathrm{cm}^{2}$ of light (10 $\mu \mathrm{M}$ of AlClPc). Cell viability is expressed as the mean \pm SD $(n=3)$. *There was a significant difference among free AlCIPc $(10 \mu \mathrm{M})$ irradiated at $140 \mathrm{~mJ} / \mathrm{cm}^{2}, 3 \mathrm{~J} / \mathrm{cm}^{2}$ and $10 \mathrm{~J} / \mathrm{cm}^{2}$ of light $(p<0.001) .{ }^{* *}$ Significant difference compared to free AlClPc irradiated at $3 \mathrm{~J} \mathrm{~cm}^{-2}(p<0.001)$.

cell viability, which suggests an efficient action of AlClPc as a photosensitiser in nanocapsules and nanospheres.

Tapajós et al. (2008) used oral carcinoma cells to evaluate AlClPc encapsulated in liposomes as an in vitro photosensitiser agent using doses of $5 \mu \mathrm{M}$ and $25 \mathrm{~J} / \mathrm{cm}^{2}$. The oral neoplasic cell destruction was predominantly started by a necrotic process after PDT. In our present study, the results obtained from these combinations of AlClPc and corresponding radiation doses were immediately lethal for the fibroblasts. The encapsulation of the sensitiser into nanoparticles improved the photoactivity of AlClPc. The encapsulated photosensitiser presented suitable biological properties in vitro and photophysical properties for its utilisation as an AlClPc delivery system in PDT.

\section{Conclusions}

The development of PLA NS, PLGA NC, PLA NC and PLA-PEG NC containing AlClPc was reported in this work with a detailed physicochemical and photophysical analysis of the different nanoparticle formulations. The incorporation of AlClPc into nanoparticles improves some parameters of the photophysical properties of AlClPc. The nanoprecipitation methodology was suitable to incorporate AlClPc into small-size nanocapsules made of different biodegradable polymers with a narrow size distribution. The advantages of AlClPc incorporation were made clear in the release studies. PLGA and PLA-PEG were able to retain AlCIPc in sink release media for more than 7 days, which resulted in a sustained release profile. The photoactivity of AlClPc was improved by encapsulation as evidenced by in vitro cell studies. Thus, the different polymeric nanoparticles studied herein have proven to be an effective method for AlClPc delivery. These particles can improve the efficiency of photodynamic therapy at very low doses and energy.

\section{Acknowledgements}

This work was supported by CNPq (481195/2011-4 project), FAPESP Projects 2006/50562-1, 2009/15363-9 and 2007/55319-0 and by the NANOBIOMG/FAPEMIG Network, Minas Gerais, Brazil. The CNPq researcher grant provided to V.C.F. Mosqueira is also acknowledged. The first author thanks UFOP and CAPES for a personal scholarship. 


\section{References}

Allison, R.R., Sibata, C.H., 2010. Oncologic photodynamic therapy photosensitizers: a clinical review. Photodiagnosis Photodyn. Ther. 7, 61-75.

Chorny, M., Fishbein, I., Danenberg, H.D., Golomb, G., 2002. Study of the drug release mechanism from tyrphostin AG-1295 loaded nanospheres by in situ and external sink methods. J. Control Release 83, 401-414.

Davda, J., Labhasetwar, V., 2002. Characterization of nanoparticle uptake by endothelial cells. Int. J. Pharm. 233, 51-59.

Dhami, S., Phillips, D., 1996. Comparison of the photophysics of an aggregation and non-aggregating aluminium phthalocyanine system incorporated into unilamellar vesicles. J. Photochem. Photobiol. A Chem. 100, 77-84.

Eaton, D.F., 1988. International union of pure and applied chemistry organic chemistry division commission on photochemistry. Reference materials for fluorescence measurements. J. Photochem. Photobiol. B. 4, 523-531.

Fessi, H., Puisieux, F., Devissaguet, J.P., Ammoury, N., Benita, S., 1989. Nanocapsule formation by interfacial polymer deposition following solvent displacement. Int. J. Pharm. 55, R1-R4.

Gref, R., Minamitake, Y., Peracchia, M.T., Trubetskoy, V., Torchilin, V., Langer, R., 1994. Biodegradable long-circulating polymeric nanospheres. Science 263 1600-1603.

Idowu, M., Nyokong, T., 2007. Photophysical and photochemical properties of zinc and aluminum phthalocyanines in the presence of magnetic fluid. J. Photochem. Photobiol. A Chem. 188, 200-206.

Kluson, P., Drobek, M., Kalaji, A., Karaskova, M., Rakusan, J., 2009. Preparation, chemical modification and absorption properties of various phthalocyanines. Res. Chem. Intermed. 35, 103-116.

Kolarova, H., Nevrelova, P., Bajgar, R., Jirova, D., Kejlova, K., Strnad, M., 2007. In vitro photodynamic therapy on melanoma cell lines with phthalocyanine. Toxicol. In Vitro 21, 249-253.

Konan, Y.N., Gurny, R., Allemann, E., 2002. State of the art in the delivery of photosensitizers for photodynamic therapy. J. Photochem. Photobiol. 66, 89106.

Konan, Y.N., Berton, M., Gurny, R., Allemann, E., 2003. Enhanced photodynamic activity of meso-tetra(4-hydroxyphenyl) porphyrin by incorporation into sub200nm nanoparticles. Eur. J. Pharm. Sci. 18, 241-249.

Kreuter, J., 1996. Nanoparticles and microparticles for drug and vaccine delivery. J. Anat. 189, 503-505.

Krishna, S., Ter Kuile, F., Supanaranond, W., Pukrittayakamee, S., Teja-Isavadharm, P., Kyle, D., White, N.J., 1993. Pharmacokinetics, efficacy and toxicity of parenteral halofantrine in uncomplicated malaria. Br. J. Clin. Pharmacol. 36, 585-591.

Labib, A., Lenaerts, V., Chouinard, F., Leroux, J.C., Ouellet, R., Van Lier, J.E., 1991. Biodegradable nanospheres containing phthalocyanines and naphthalocyanines for targeted photodynamic tumor therapy. Pharm. Res. 8, 1027-1031.

Legrand, P., Barrat, G., Mosqueira, V.C.F., Fessi, H., Devissaguet, J.P., 1999. Polymeric nanocapsules as drug delivery systems: as review. Pharm. Sci. 9, 411-418.

Legrand, P., Lesieur, S., Bochot, A., Gref, R., Raatjes, W., Barrat, G., Vauthier, C., 2007. Influence of polymer behaviour in organic solution on the production of polylactide nanoparticles by nanoprecipitation. Int. J. Pharm. 344, 33-43.

Leite, E.A., Vilela, J.M.C., Mosqueira, V.C.F., Andrade, M.S., 2005. Poly-caprolactone nanocapsules morphological features by atomic force microscopy. Microsc. Microanal. 11, 48-51.

Maeda, H., 2010. Tumor-selective delivery of macromolecular drugs via the EPR effect: Background and future prospects. Bioconjug. Chem. 21, 797-802.
Mosmann, T., 1983. Rapid colorimetric assay for cellular growth and survival: application to proliferation and cytotoxicity assays. J. Immunol. Methods 65 55-63.

Mosqueira, V.C.F., Legrand, P., Pinto-Alphandary, H., Puisieux, F., Barratt, G., 2000. Poly(D, L-Lactide) nanocapsules prepared by a solvent displacement process: Influence of the composition on physicochemical and structural properties. J. Pharm. Sci. 89, 614-626.

Mosqueira, V.C., Legrand, P., Gulik, A., Bourdon, O., Gref, R., Labarre, D., Barratt, G. 2001a. Relationship between complement activation, cellular uptake and surface physicochemical aspects of novel PEG-modified nanocapsules. Biomaterials 22, 2967-2979.

Mosqueira, V.C.F., Legrand, P., Morgat, J., Vert, M., Mysiakine, E., Gref, R., Devissaguet, J.-P., Barratt, G., 2001b. Biodistribution of long-circulating PEGgrafted nanocapsules in mice: effects of PEG chain length and density. Pharm. Res. 18, 1411-1419.

Moura-Siqueira, M.P., Primo, F.L., Peti, A.P.F., Tedesco, A.C., 2010. Validated spectrophotometric and spectrofluorimetric methods for determination of chloroaluminum phthalocyanine in nanocarriers. Pharmazie 65, 9-14.

Nunes, S.M.T., Sguilla, F.S., Tedesco, A.C., 2004. Photophysical studies of zinc phthalocyanine and chloroaluminum phthalocyanine incorporated into liposomes in the presence of additives. Braz. J. Med. Biol. Res. 37, 273-284.

Oliveira, D.M., MacAroff, P.P., Ribeiro, K.F., Lacava, Z.G.M., Azevedo, R.B., Lima, E.C.D. Morais, P.C., Tedesco, A.C., 2005. Studies of zinc phthalocyanine/magnetic fluid complex as a bifunctional agent for cancer treatment. J. Magn. Magn. Mater. 289, 476-479.

Oliveira, L.T., Garcia, G.M. Kano, E.K. Tedesco, A.C, Mosqueira, V.C.F., 2011. HPLCFLD methods to quantify chloroaluminum phthalocyanine in nanoparticles, plasma and tissue: application in pharmacokinetic and biodistribution studies. J. Pharm. Biomed. Anal. 1, 70-77.

Rocha, M.S.T., Lucci, C.M., Longo, J.P.F., Galera, P.D., Simioni, A.R., Lacava, Z.G.M., Tedesco, A.C., Azevedo, R.B., 2012. Aluminum-chloride-phthalocyanine encapsulated in liposomes: activity against naturally occurring dog breast cancer cells. J. Biomed. Nanotechnol. 8, 251-257.

Rodrigues, G.B., Primo, F.L., Tedesco, A.C., Braga, G.U.L., 2012. In vitro photodynamic inactivation of cryptococcus neoformans melanized cells with chloroaluminum phthalocyanine nanoemulsion. Photochem. Photobiol. 88, 440-447.

Sibata, M.N., Tedesco, A.C., Marchetti, J.M., 2004. Photophysical and photophysical studies of Zinc (II) phthalocyanine in long time circulation micelles for photodynamic therapy use. Eur. J. Pharm. Sci. 23, 131-138.

Silva, A.R.A., Simioni, A.R., Tedesco, A.C., 2011. Photophysical and complexation studies of chloro-aluminum phthalocyanine with beta-cyclodextrin and hydroxypropyl-beta-cyclodextrin. J. Nanosci. Nanotechnol. 11, 4046-4055.

Soppimath, K.S., Aminabhaui, T.M., Kulkarni, A.R., Rudzinski, W.E., 2001. Biodegradable polymeric nanoparticle as delivery devices. J. Control Release 70, $1-20$.

Tapajós, E.C.C., Longo, J.P., Simioni, A.R., Lacava, Z.G.M., Santos, M.F.M.A., Morais, P.C., Tedesco, A.C. Azevedo, R.B., 2008. In vitro photodynamic therapy on human oral keratinocytes using chloroaluminum-phthalocyanine. Oral Oncol. 44, 1073-1079.

Tedesco, A.C., Rotta, J.C.G., Lunardi, C.N., 2003. Nitric oxide release from the $S$ nitrosothiol zinc phthalocyanine complex by flash photolysis. Braz. J. Medical Biol. Res. 36, 587-594.

Vila, A., Gill, H., McCallion, O., Alonso, M.J., 2004. Transport of PLA-PEG particles across the nasal mucosa: effect of particle size and PEG coating density. J. Control. Release. 98, 231-244. 